Anror phous $\mathrm{TI}$ 1- $\mathrm{xCuxCl}$ sol i $d$ sol ut $i$ on and thei $r$ crystal I i zati on st udi ed by opt i cal absor pt i on spect r oscopy

\begin{tabular}{|l|l|}
\hline 著者 & KONDO S, TANAKA H, SAl TO T \\
\hline $\begin{array}{l}\text { j our nal or } \\
\text { publ i cat i on t i t l e }\end{array}$ & Nat er i al s Sci ence and Engi neeri ng B \\
\hline vol une & 78 \\
\hline number & 2 \\
\hline page range & $90-95$ \\
\hline year & $2000-12$ \\
\hline URL & ht t p: //hdl . handl e. net /10098/1544 \\
\hline
\end{tabular}




\title{
Amorphous $\mathrm{Tl}_{1-x} \mathrm{Cu}_{x} \mathrm{Cl}$ solid solution and their crystallization studied by optical absorption spectroscopy
}

\author{
S. Kondo , H. Tanaka, T. Saito \\ Research Center for Development of Far-Infrared Region, Fukui University, Bunkyo, Fukui 910-8507, Japan
}

\begin{abstract}
Abstrace
Amorphous solid solutions are obtainable for the binary $\mathrm{Tl}_{1-x} \mathrm{Cu}_{x} \mathrm{Cl}$ system by means of two-sources evaporation onto $77 \mathrm{~K}$ substrates. Their annealing behavior has been studied for a film with $x \sim 0.4$ by optical absorption spectroscopy. In the as-prepared film an appreciable amount of $\mathrm{Cu} 3 \mathrm{~d}$ states is localized with the energies lying above the upper valence bands. On annealing the film, $\mathrm{Cu}^{+}$ions migrate to form clusters in the amorphous environment, with their size depending on the annealing temperature. The lowest annealing temperature at which the clusters are formed lies near the crystallization temperature of amorphous $\mathrm{CuCl}$. On further annealing at higher temperatures, the clusters grow until the amorphous environment has a particular atomic composition ratio suggestive of a new, novel compound, which exhibits a well defined crystallization temperature, $300 \mathrm{~K}$.
\end{abstract}

Keywords: $\mathrm{T} l_{-,} \mathrm{Cu} \mathrm{C}_{x} \mathrm{C}$; Crystalization; Optical absorption spectroscopy

\section{Introduction}

A lot of metall halides such as silver [1], copper [2], thallium [3], lead [4] or cadmium [5] halides have been shown to be amorphized by quench deposition yielding film samples. The amorphous films exhibit excellent transmittance below the absorption edge and have well-defined, characteristic crystallization temperature at which the optical absorption spectrum changes distinctly. In particular, crystallization of the amorphous films of mixed metal halides produces small clusters or nanocrystals. For instance, we recently obtained [6] $\mathrm{CdI}_{2}$ films in which stable two-molecule $\mathrm{Bil}_{3}$ clusters were densely monodispersed up to $10 \mathrm{~mol} \%$ of $\mathrm{Bil}_{3}$ concentration. The clusters were stoichiometrically embedded in the $\mathrm{CdI}_{2}$ crystal matrix. It was also possible to disperse $\mathrm{CuCl}$ clusters in crystalline $\mathrm{PbCl}_{2}$ films at high density $(\sim 3 \mathrm{~mol} \%)[7]$. In these studies the amorphous films of the metal halides heavily doped with foreign metal ions were obtainable by evaporating the mixture of two metal halides (host and guest metal halides) onto cooled substrates; in the amorphous surroundings the cations of the metal halides were replaceable by foreign metal ions up to appreciable concentrations despite the difference in the valency between the guest and host cations. The crystallization of the amorphous films yielded small clusters or nanocrystals of the guest-metal halide embedded in the crystalline matrix of the host-metal halide. These results suggest that amorphization and subsequent crystallization of the mixture of metal halides provide a new method for obtaining small clusters or nanocrystals.

In the present work a similar study was carried out on the binary $\mathrm{TlCl}-\mathrm{CuCl}$ system to investigate the properties of amorphization and crystallization in the mixed system of equal cation valencies. The amorphization was achieved by using an improved setup, which, was developed to prepare amorphous films of various mixed systems. It was shown that $\mathrm{TlCl}$ and $\mathrm{CuCl}$ can be made amorphous solid solution up to a significant concentration ratio because of their equal cation valencies. The annealing of the amorphous solid solution yielded $\mathrm{CuCl}$ clusters densely dispersed in a new, amorphous or crystalline environment with their size depending on the annealing temperature. 


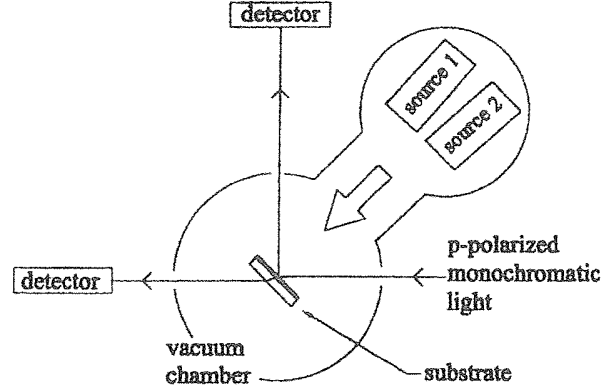

Fig. 1. A schematic view of experimental configuration illustrating preparation of two-sources evaporated amorphous fllms of mixed metal halides and in situ UV absorption measurement of the films.

\section{Expermentals}

Fig. 1 shows a geometrical and optical configuration of the experimental setup used. It is equipped for preparing quench-deposited amorphous films of mixed metal halides and for studying their crystallization behavior in situ by optical absorption spectroscopy. Amorphous films of the binary $\mathrm{TICl}-\mathrm{CuCl}$ system were obtained by means of a two-sources (source 1 and source 2) evaporation onto a silica-glass substrate cooled to 77 $\mathbb{K}$ in a vacuum of about $9 \times 10^{-6} \mathrm{~Pa}$, using a pair of evaporators. The body of the evaporators was made up of a copper block, in which a cylindrical cell and two cartitidge heaters were embedded. A powder reagent put in the cell was vaporized by the heaters in a controlled manner. The use of the two evaporators in a pair enabled us to obtain amorphous films of mixed $\mathrm{TlCl}-\mathrm{CuCl}$ films with any composition ratios. Details including the construction of the evaporators will be reported elsewhere. Optical properties of amorphous films and their crystallization behavior were investigated in situ by UV absorption spectroscopy, using an improved double beam method described in [6]. The method is based on simultaneous measurements of transmittance and reflectance from which accurate optical densities of the films can be determined.

\section{Residilis}

Fig. 2 shows the absorption spectra at $77 \mathrm{~K}$ of a quench-deposited $\mathrm{Tl}_{1-x} \mathrm{Cu}_{x} \mathrm{Cl}$ film with $x \sim 0.4$. Spectrum I was first measured for the as-prepared fim. Then the film was subjected to various annealing cycles to observe irreducible change in the spectrum at $77 \mathrm{~K}$ with increasing annealing temperature. Heating and cooling in each annealing cycle were performed at rates of 1 and $10 \mathrm{~K} \mathrm{~min}^{-1}$, respectively. The annealing temperature, $T_{\mathrm{a}}$ $(K)$, and annealing time, $t_{\mathrm{a}}(\mathrm{min})$, for each spectrum labeled with the number, $n$, in the figure are, in terms of $n\left(T_{\mathrm{a}}, t_{\mathrm{a}}\right)$, as follows: $2(100,1), 3(120,1), 4(140,1), 5$
$(160,1), 6(180,1), 7(200,1), 8(220,1), 9(240,1), 10$ $(260,1), 11(280,1), 12(300,1), 13(300,10), 14(300$, $180)$ and $15(360,1)$.

The spectra labeled 1 to 12 in Fig. 2 are all characterized by nearly the same spectral feature in the photon energy region above $4 \mathrm{eV}$ exhibiting a monotonous broad band around $5.5 \mathrm{eV}$; annealing of the film up to $T_{\mathrm{a}}=300 \mathrm{~K}$ results in almost no spectral change in this photon energy region. On annealing for $10 \mathrm{~min}$ at 300 $\mathrm{K}$, however, an abrupt change occurred in the spectral shape as seen from spectrum 13, giving rise to several fine, but weak, structures (at 4.1, 4.6, 5.5 and $5.8 \mathrm{eV}$ ). These structures were stable and unchanged during a further annealing period, $t_{\mathrm{a}}=180 \mathrm{~min}$, at $T_{\mathrm{a}}=300 \mathrm{~K}$ (spectrum 14) and even for a higher annealing temperature $T_{\mathrm{a}}=360 \mathrm{~K}$ (spectrum 15 ).

In the photon energy region below $4 \mathrm{eV}$, on the other hand, there occurs gradual change in the spectral structure with increasing annealing temperature even below $300 \mathrm{~K}$. Fig. 3 illustrates this photon energy region in a

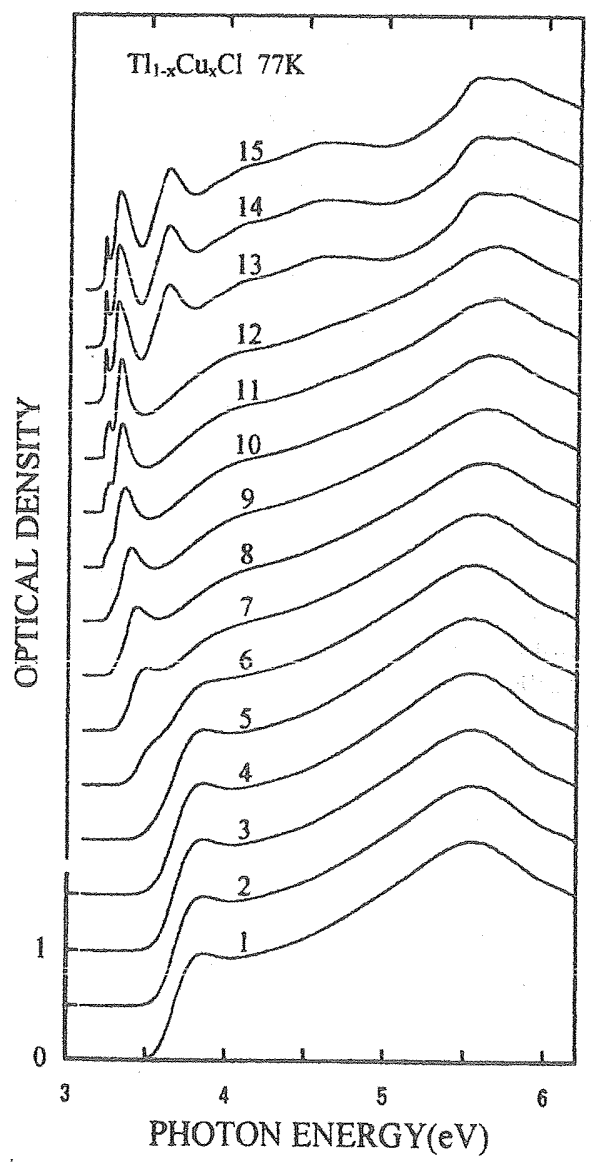

Fig. 2. The effect of annealing on optical absorption of a $\mathrm{Tl}_{1}$ $x \mathrm{Cu}, \mathrm{Cl}(x \sim 0.4)$ film prepared by means of two-sources evaporation onto $77 \mathrm{~K}$ substrate. The absorption spectra are measured at $77 \mathrm{k}$ for increasing annealing temperatures. 
magnified scale, where spectra $1-4$ and spectra 14 and 15 are omitted because their shapes are entirely the same as spectrum 5 and spectrum 13, respectively. As seen from the figurc, a new absorption band shows up around $3.5 \mathrm{eV}$ for $T_{\mathrm{a}}=180 \mathrm{~K}$ (spectrum 6 ). As the annealing temperature is increased, it becomes prominent and shifts to low energies exhibiting two absorption bands, around 3.24 and $3.32 \mathrm{eV}$ for $\mathrm{T}_{\mathrm{a}}=300 \mathrm{~K}$ $\left(i_{\mathrm{a}}=1 \mathrm{~min}\right.$, spectrum 12), which correspond to the CuCl $Z_{3}$ and $Z_{1,2}$ excitons [8], respectively. As compared with this gradual change in the spectrum with increasing $T_{\mathrm{a}}$ up to $300 \mathrm{~K}$, further annealing at $300 \mathrm{~K}$, but only for $10 \mathrm{~min}$, of the film (spectrum 13) gave rise to an abrupt spectral change characterized by the sharpened $\mathbb{Z}_{3}$ exciton peak and a new, strong absorption band appearing around $3.6 \mathrm{eV}$ with a weak shoulder at about $3.53 \mathrm{eV}$. These features of spectrum 13 were shown to remain unchanged for further annealings (spectra 14 and 15 in Fig. 2).

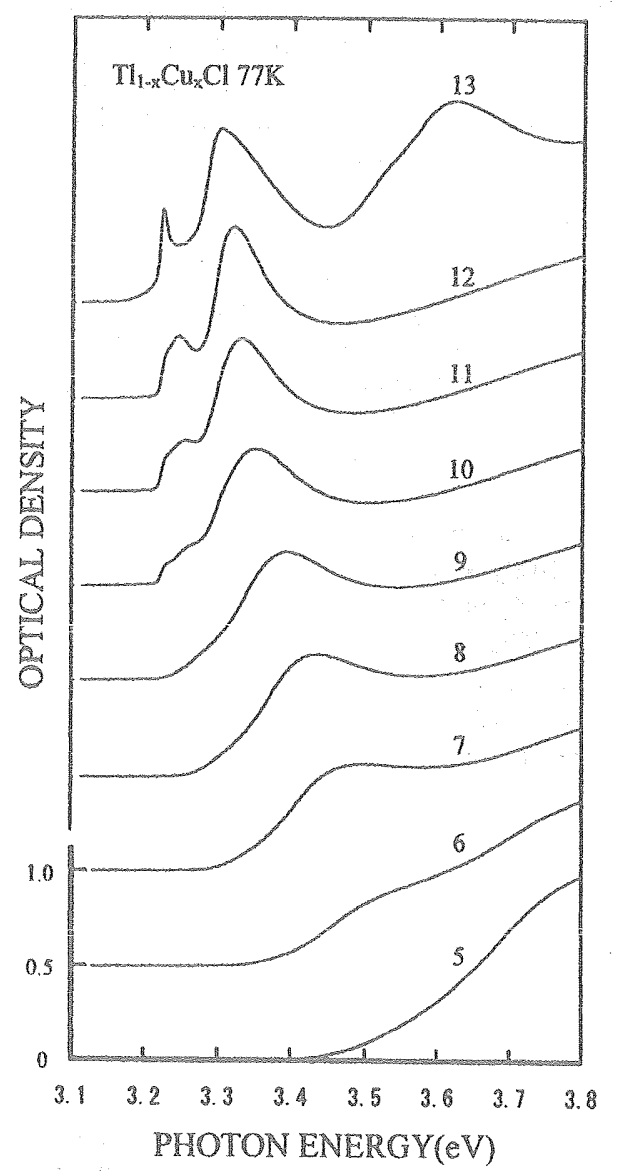

Tig. 3. Spectra 513 in Fig. 2 are replotted in a magnified scale in the region near the fundamental edges.

\section{费. Discussion}

As mentioned in Section 1, amorphous films of metal halides including $\mathrm{TICl}$ and $\mathrm{CuCl}$ have well-defined crystallization temperatures $(98 \mathbb{K}$ for $\mathrm{TlCl}[3], 177 \mathrm{~K}$ for CuCl [2]), at which there occurs distinct change in their absorption spectrum. In the present experiments, we found that such a characteristic change of spectrum also occurs in the $\mathrm{Tl}_{\mathrm{I}_{-x}} \mathrm{Cu}_{x} \mathrm{Cl}$ fim for $\mathrm{T}_{\mathrm{a}}=300 \mathrm{~K}$, seen from the distinct difference between spectra 12 and 13 Fig. 2. It may be supposed, therefore, that the film is in amorphous state below $300 \mathrm{~K}$ and crystallization of the film starts to occur at $T_{a}=300 \mathbb{K}$ and is completed within $10 \mathrm{~min}$ at that temperature. The crystalline nature for spectrum 13 is read by the fine structures appearing above $4 \mathrm{eV}$, which are reminiscent of van Hove singularities of the associated energy band. The completion of the crystallization (within $10 \mathrm{~min}$ at 300 $\mathbb{K}$ ) is evidenced by the fact that no further spectral change occurred during further amealing at $300 \mathrm{~K}$ or even at higher annealing temperatures ( $\leq 360 \mathrm{~K}$ ).

To elucidate characteristics of the "amorphous' $\mathrm{Tl}_{1-x} \mathrm{Cu}_{x} \mathrm{Cl}$ flm, we will first mention the absorption spectra of $\mathrm{TlCl}[3]$ and $\mathrm{CuCl}[2]$ films in terms of anorphous versus crystalline. Curves a and $\mathrm{c}$ in Fig. $4 \mathrm{a}$ (TICl) and Fig. $4 \mathrm{~b}(\mathrm{CuCl})$ are the spectra of the amorphous and crystalline (crystallized) flins in the exciton energy region, respectively. For comparison spectra, 1 and 12 of the $\mathrm{T}_{1-x} \mathrm{Cu}_{x} \mathrm{Cl}$ fim are also ploted in Fig. 4c. The crystaline films exhibit a sharp exciton peak at $3.49 \mathrm{eV}$ for TICl and two very sharp ones at $3.224\left(Z_{3}\right)$ and $3.29 \mathrm{eV}\left(Z_{12}\right)$ for $\mathrm{CuCl}$ (the non-zero optical densities in CuCl in the otherwise transparent region below $3.2 \mathrm{eV}$ are due to devitrification during crystallization and subsequent crystal growth). Although there is no indication of exciton transitions in the amorphous film of $\mathrm{TICl}$, discernible exciton bands are observed for the amorphous $\mathrm{CuCl}$ film due to a very small exciton radius $\left(0.68 \mathrm{~mm}\right.$ for $\mathbb{Z}_{3}$ [9], which is so small that the exciton is able to exist within the extent of the shortrange order [2]). Spectrum 1 of the $\mathrm{Tl}_{1-x} \mathrm{Cu}, \mathrm{Cl}$ film is very similar in feature near the absorption edge to curve a of TICl but with the somewhat red-shifted (by about $0.3 \mathrm{eV}$ ) optical energy gap and exhibits no sign of $\mathrm{CuCl}$ exciton absorption despite the considerable $\mathrm{CuCl}$ concentration in the fim. This suggests that the $\mathrm{Cu}$ atoms in the as-prepared $\mathrm{N}_{1-x} \mathrm{Cu}$ Cl film are substituted for Til atoms in the $\mathrm{TlCl}$ amorphous network, thus forming amorphous solid solution of a $\mathrm{TICl}$ base.

It is well known that in amorphous semiconductors a certain amount of extended states near the band edges is localized due to amorphization-induced structural disorder. As to amorphous $\mathrm{TlCl}$, whose absorption spectrum near the fundamental edge is characterized by the missing excitonic absorption and blue-shifted (by about $0.4 \mathrm{eV}$ ) optical energy gap as shown in Fig. $4 \mathrm{a}$, it 


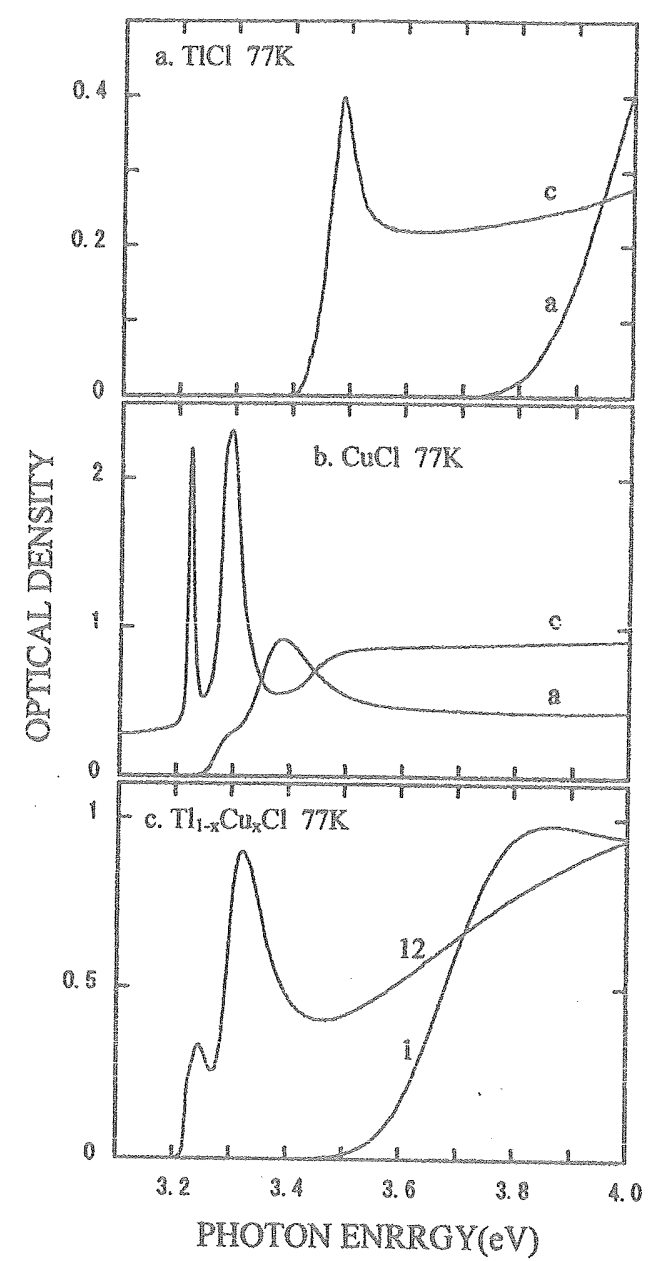

Fig. 4. Absorption spectra of (a) TlCl, and (b) CuCl measured at 77 $\mathrm{K}$ for the amorphous (curves a) and crystalline (curves c) nims. For comparison, spectra 1 and 12 of (c) $\mathrm{Tl}_{1}{ }_{x} \mathrm{Cu} . \mathrm{Cl}(x+0.4)$ are also presented.

was necessary to assume that a considerable amount of the uppermost valence band coming from the Tl-6s state and the lowemost conduction band from the Tl-6 $p_{1 / 2}$ states were localized by amorphization [10], in order to explain the characteristic absorption spectrum. In the case of amorphous $\mathrm{Tl}_{1-x} \mathrm{Cu}_{x} \mathrm{Cl}$ solid solution, further localization of electronic states is expected to occur due to the effect of chemical disorder. If $\mathrm{Cu}$ atoms are substiuted for $\mathrm{Tl}$ atoms at a high density in the $\mathrm{TlCl}$ crystal matrix, the $\mathrm{Cu}-3 \mathrm{~d}$ states may contribute to upper valence bands (in $\mathrm{CuCl}$, the $\mathrm{Cu}-3 \mathrm{~d}$ states indeed contribute to upper valence bands [11]). It is probable, therefore, that an appreciable amount of the $\mathrm{Cu}-3 \mathrm{~d}$ states are localized in the amorphous $\mathrm{Tl}_{1-x} \mathrm{Cu}_{x} \mathrm{Cl}$ solid solution. This may account for the smaller optical energy gap for the amorphous
$\mathrm{Tl}_{1}, \mathrm{Cu}_{x} \mathrm{Cl}$ solid solution than for amorphous $\mathrm{TICl}$ The strong absorption appearing as a broad band around $3.8 \mathrm{eV}$ for the former may be due to a high density of the localized Cu-3d states.

The crystallization temperature of the amorohous $\mathrm{Tl}_{1-x} \mathrm{Cu} x \mathrm{Cl}$ solid solution, $300 \mathrm{~K}$, is much higher than those of both amorphous films of TIC $(98 \mathrm{~K})$ and $\mathrm{CuCl}$ (177 K). This exemplifies that the chemical disorder has the effect of increasing the crystallization temperature. Such increased crystallization temperatures due to chemical disorder were previously observed for amorphous $\mathrm{TlCl}_{1-x} \mathrm{Br}_{x}$ solid solution (e.g. $137 \mathrm{~K}$ for $x=0.4$ as compared with 98 and $95 \mathrm{~K}$ for amorphous TICl and THBr, respectively [121). In the present experiment, however, the situation is somewhat different. As seen from Fig. 3 , on annealing the amorphous $\mathrm{T}_{1}, \mathrm{Cu}_{r} \mathrm{Cl}$ solid solution, a new absorption band begins to show up around $3.5 \mathrm{eV}$ for $\mathrm{T}_{\mathrm{a}}=180 \mathrm{~K}$ (spectrum 6). The band may be due to formation of $\mathrm{CuCl}$ clusters because it grows to the $\mathrm{CuCl} \mathbb{Z}_{3}$ and $\mathbb{Z}_{1,2}$ exciton peaks with increasing $T_{a}$ (e.g. spectrum 12). It can be stated, therefore, that the $\mathrm{Cu}^{+}$ions begin to migrate to form clusters in the amorphous environment at annealing temperatures near the crystallization temperature of amorphous $\mathrm{CuCl}$. Such a clustering behavior is in great contrast to that observed in the system of heavily $\mathrm{Cu}^{+}$-doped amorphous $\mathrm{PbCl}_{2}$, where crystallization of the host $\mathrm{PbCl}_{2}$ accompanies the formation of $\mathrm{CuCl}$ cinsters, which are necessarily embedded in the crys. talline matrix of $\mathrm{PbCl}_{2}$. In the present $\mathrm{Tl}_{1-x} \mathrm{Cu} \times \mathrm{Cl}$ system, however, the CuCl clusters are considered to be embedued in the amorphous matrix.

It was shown in [2] that the excitons in amorphous $\mathrm{CuCl}$ are 'confined' within a size defined by the shortrange order in the amorphous network. The corresponding exciton energies ( 3.28 and $3.38 \mathrm{eV}$ for $\mathbb{Z}_{3}$ and $Z_{1,2}$, respectively as shown in $F i g .4 b$ ) were nearly cqual to those of $\mathbb{Z}_{3}$ and $\mathbb{Z}_{1,2}$ of spectrum $9\left(T_{2}=240 \mathrm{~K}\right)$ in Fig. 3. Therefore, amnealing of the amorphous $\mathrm{Tl}_{1-x} \mathrm{Cu}_{x} \mathrm{Cl}$ solid solution at temperatures below 240 $\mathbb{K}$ (but above $177 \mathrm{~K}$ ) yielded $\mathrm{CuCl}$ clusters of a size smaller than the extent of the short-range order of the $\mathrm{CuCl}$ amorphous network, as suggested by the corresponding blue-shifted absorption band observed for spectra $6-8$. Further annealings at temperatures above $240 \mathrm{~K}$ resulted in the growth of the $\mathrm{CuCl}$ clusters (spectra $10\left(T_{\mathrm{a}}=260 \mathrm{~K}\right)$ to $\left.12\left(T_{\mathrm{a}}=300 \mathrm{~K}\right)\right)$ which were embedded in the $\mathrm{Tl}_{1-x} \mathrm{Cu}_{x} \mathrm{Cl}$ amorphous matrix, and the crystallization of the amorphous matrix occurred for annealing at $300 \mathrm{~K}$ and is completed within $10 \mathrm{~min}$ (spectrum 13) as mentioned above. After the crystallization, the $\mathbb{Z}_{3}$ exciton absorption exhibited a sharp peak at $3.224 \mathrm{eV}$, which coincided with the $\mathbb{Z}_{3}$ exciton peak energy of the crystalline $\mathrm{CuCl}$ fim in Fig. $4 b$. 
Spectrum 13 exhibited neither discernible structure reminiscent of TICl exciton absorption (at $3.49 \mathrm{eV}$ ) nor enhanced absorption of the $\mathrm{CuCl} Z_{3}$ and $Z_{1,2}$ excitons (compared with that of spectrum 12); instead, the spectrum was characterized by a prominent absorption band around $3.6 \mathrm{eV}$ (with a shoulder at about $3.53 \mathrm{cV}$ ). This suggests that the crystalline matrix in which the $\mathrm{CuCl}$ clusters were embedded was made of a new, novel compound, and negated the possibility of an eutectic product (mixture of $\mathrm{TlCl}$ and CuCl crystallites) for the crystalline matrix. The absorption intensity of the $\mathrm{CuCl} \mathbb{Z}_{3}$ and $\mathbb{Z}_{1,2}$ excitons seems to be saturated before the crystallization of the amorphous matrix. In fact, the integrated absorption intensity in the region $3.2-3.55 \mathrm{eV}$ was shown to be entirely the same (within the experimental errors) for spectra $11\left(T_{a}=280 \mathrm{~K}\right)$ and 12 . This indicates that a certain concentration ratio of the $\mathrm{Cu}$ atoms to the TI atorns, appropriate to the molecular formula of the new compound, is reached in the amorphous matrix at $T_{\mathrm{a}}=280 \mathrm{~K}$, owing to the decrease of the $\mathrm{Cu}$ atoms due to the growth of the CuCl clusters (the somewhat smaller band widths for $Z_{3}$ and $Z_{1,2}$ of spectrum 12 than of spectrum 11 may be due to a narrowed sizedistribution of the $\mathrm{CuCl}$ clusters due to annealing). Therefore, the annealing temperature ' $300 \mathbb{K}$ ' for spectrum 13 is considered to be the crystallization temperature for the amorphous phase of the new compound -... the amorphous thm of the new com pound has a welldefned, characteristic crystallization temperature, $300 \mathrm{~K}$, at which the absorption spectrum changes distinctly, similar to other metal halides mentioned in the first paragraph in Section 1.

It is noted that the $\mathrm{CuCl}$ microcrystals embedded in the amorphous matrix exhibit the much larger band width for $\mathbb{Z}_{3}$ excitons than the case of the crystalline matrix, as seen from the comparison of spectra 12 and 13. A plausible explanation for this might be given in terms of a size distribution of the $\mathrm{CuCl}$ microcrystals. If this is the case, however, the band width for $\mathbb{Z}_{1,2}$ relative to the enhanced band width for $\mathbb{Z}_{3}$ in spectrum 12 would be too large to be favorably compared with the observed $\mathbb{Z}_{1,2}$ band width of spectrum 12, since the size effect on exciton energies should be stronger for $\mathbb{Z}_{1,2}$ than for $\mathbb{Z}_{3}$ because of the larger exciton radius for the former. Therefore, another explanation should be given. One of the possible explanations riay be in terms of 'effective strains'. It is considered that the boundaries between the amorphous matrix and the individual $\mathrm{CuCl}$ microcrystals embedded therein are less well-defned than the case of the crystalline matrix. Therefore, the exciton wave functions for the former may be under 'effective strains' near the boundaries. Such strains may cause various magnitudes of the shift of the exciton energy according to the different modes and different magni- tudes of the strains. According to [13], the effect of strains on bulk $\mathrm{CuCl}$ excitons is stronger for $\mathbb{Z}_{3}$ than for $\mathbb{Z}_{1,2}$ as far as the shift of the exciton energies are concerned. These considerations are favorable for explaining the enhanced $\mathbb{Z}_{3}$ band widh under consideration.

\section{Conch}

The two compounds $\mathrm{TlCl}$ and $\mathrm{CuCl}$, though crystallize in different crystal structures, can be rendered amorphous solid solution with high mixing ratios by means of two-sources evaporation onto $77 \mathrm{~K}$ substrates, yielding fim samples. Annealing behaviors of a representarive fim of the amorohous solid solution, $\mathrm{Tl}_{1-x} \mathrm{Cr}_{x} \mathrm{Cl}$ with $x \sim 0.4$, was studied by optical absorption spectroscopy. The absorption spectrum of the as-prepared (before annealing) film was character. ized by enhanced, non-excitonic absorption near the fundamental edge, due to contribution of localized $\mathrm{Cu}$ $3 \mathrm{~d}$-states. When the film was annealed at temperatures near the crystallization temperature (177 K) of amorphous $\mathrm{CuCl}$, new absorption band appeared around $3.5 \mathrm{eV}$ due to formation of $\mathrm{CuCl}$ clusters in the amorphous environment. On further amnealing at higher temperatures, the band grew to well resolved $\mathbb{Z}_{3}$ and $\mathbb{Z}_{1,2}$ bands indicating the formation of $\mathrm{CuCl}$ microcrystals, with their total integrated absorption intensity showing the tendency to saturate at the annealing temperature about $280 \mathrm{~K}$. As a result, the amorphous environment had a particular atomic conposition of $\mathrm{Tl}$ and $\mathrm{Cu}$ suggestive of a novel compound which had a well defined, characteristic crystallization temperature, $300 \mathrm{~K}$. The CuCl microcrystals, embedded in the amorphous matrix of the novel compound exhibited a large band width for $\mathbb{Z}_{3}$, probably due to 'effective strains' near the microcrystal surfaces.

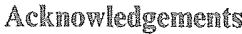

This work was partly supported by The Hokuriku Industrial Advancement Center and partly by the grant-in-aid for Scientific Research from the Ministry of Education, Science, Sports and Culure, Japan.

\section{Meferences}

[1] H.G. Gotwald, K.G. Weil, Der. Bunsenges Phys, Chem. 92 (1988) 60.

[2] S. Kondo, T. Seki, Mater. Sci. Eng. D 15 (1992) 133.

[3] S. Kondo, T. Itoh, T. Saito, M. Mekata, Solid State Commun. 78 (1991) 557 . 
41 S. Kondo, T. Sakai, 琵. Tanaka, T. Saito, Phys. Rey. B 58 (1998) 11401 .

[5] S. Kondo, S. Ragawa, T. Saito, Phys. Status Solidi B 154 (1996) 583.

[6] S. Kondo, T. Suzuki, T. Saito, J. Phys. D 31 (1998) 2733.

[7] S. Kondo, N. Tanaka, T. Saío, J. Phys. D 33 (2000) 327.

[8] M. Cardona, Phys. Rev. 129 (1963) 69.

[9] B. Honerlage, C. Klngshim, J.B. Grun, Phys. Status Solid: B $78(1976) 599$.
[10] S. Kondo, H. Tamaka, T. Saito, J. Phys. Condens. Mater. It (1999) 8155

[11] K. Shindo, A. Morita, H. Kamimura, J. Phys. Soc. Jpn. 20 (1965) 2054

[12] S. Kondo, N. Yamagishi, T. Saito, J. Phys. Soc, Jpn. 67 (1998) 353 .

[13] T. Koda, T. Murahashi, T. Mtani, Phys. Rev. B 5 (1972) 705 . 